\title{
The Case for Technobiology: A Complement to Biotechnology
}

\author{
James M TIEN \\ College of Engineering, University of Miami, Coral Gables, Florida 33124, USA \\ Pascal J GOLDSCHMIDT-CLERMONT \\ Miller School of Medicine, University of Miami, Coral Gables, Florida 33124, USA
}

\begin{abstract}
Engineering has and will continue to have a critical impact on healthcare; the application of technology-based techniques to biological problems can be defined to be technobiology — it can be considered to be the complement to biotechnology, an all too encompassing and overused term. If technobiology can be thus defined, then biotechnology can be conversely defined to be the application of biology-based techniques to technical problems; as a consequence, the two complementary terms, together, can cover the increasingly expanding and complex world where technology and biology intersect. In addition to detailing the scope of technobiology, this paper expands on the technobiology approach of service systems engineering to the development of a healthcare service system that is integrated, adaptive and evidence-based; focuses on a range of example applications in regard to the technobiology areas of information, instrumentation, and insertion; underscores, as an example, the collaborative technobiology efforts between the College of Engineering and the Miller School of Medicine at the University of Miami; and concludes with some additional insights.
\end{abstract}

Keywords biotechnology; technobiology; goods; services; servgoods; Big Data; decision analytics; value chain; supply chain; demand chain; system integration; adaptive services; mass production; mass customization; Internet of Things; Internet of connected Servgoods

\section{Background}

During the time surrounding World War I, the need for agrarian reform in famine-stricken Central Europe was a critical problem; for an obscure Hungarian engineer, Karloly Ereky ${ }^{[1]}$, the term "biotechnologie" elucidated the process by which organisms could be used to create commercial products from raw materials, thereby reorganizing small-scale farms into large-scale agricultural operations. For example, Ereky industrialized pig production by using sugar beets as a source of nutrition. Based upon his pioneering research on chlorophyll, Ereky also developed a commercial product using leaf protein in livestock feed. Thus, through the application of biology (including biochemistry) to technology nearly a century ago, Ereky established a new biotechnology paradigm in science and engineering. Today, we have, as examples, appropriately identified neural networks (i.e., the application of how neurons network and function to help model complex relationships between inputs and outputs or to find patterns in data), genetic algorithms (i.e., the application of natural selection rules to evolutionary programs and processes), and systems biology (i.e., the application of how our body functions to help design

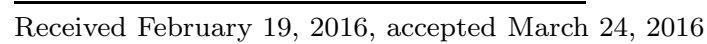


more resilient man-made systems that could function in an integrative, adaptive and intelligent manner) as breakthroughs in biotechnology or the application of biology to technology. Alternative terms for biotechnology include biomimetics (whereby new technologies inspired by biological solutions at both the macro- and nano-scales), biomimicry (whereby self-healing and self-assembling abilities are mimicked), and bionics (whereby intelligently powered exoskeleton devices can be employed to enhance the strength, mobility, and endurance of soldiers and paraplegics).

Unfortunately, the term "biotechnology" has been overused to describe all activities that include elements of biology (including healthcare) and elements of technology (including engineering). If the term biotechnology is about applying biology to technology, then we need a corresponding or complementary term that is about applying technology to biology. By definition, healthcare refers to the treatment and management of illness, while engineering refers to the application of technical, scientific and mathematical knowledge to help design, implement and upgrade products (including devices and structures), services (including co-produced products and processes), and systems (including integrated products and services). Clearly, engineering healthcare is about applying technology to biology; more specifically, employing a similar Ereky logic, it is about "technobiology". The technolobiology examples listed in Table 1 highlight their disciplinary focus, including biomedical, chemical, electrical, environmental, industrial, material and mechanical devices, approaches, and processes. Indeed, as briefly identified in Table 1, a short list of such technobiology applications include: Bioimaging techniques; neurostimulators; regenerative methods such as tissue engineered induced pluripotent stem (iPS) cells for replacing damaged skin, muscle, and bone; enhanced DNA sequencing with chips and quantum dots; genetic tests for debilitating diseases; antimicrobial nanoparticle dressings for infected wounds; robotic, bionic and prosthetic devices for the mobility impaired; replacement body parts; new drugs through bioinformatics; bioelectronics; adaptive design of experiments; preventive, concierge and personalized care; time-delayed, drug delivery methods; and nanoparticles for cancer treatments. The fact remains: Technology has affected every aspect of our lives, from housing to finance, to manufacturing, to learning, to wellness and, increasingly, to our health.

As indicated earlier, engineering is about developing new and better products, services and systems. When engineering a service (including healthcare), the concept of co-production is paramount. Thus, co-production in technobiology strives to maximize the abilities of the patient and the provider to achieve a common goal: Improved health. As such, it at once can enhance outcome of treatment provisions, while refocusing the essence of a healthcare system from provider-directed to a provider-patient partnership. Typically, the role of the patient in improving outcomes has been reflected through treatment compliance, follow-up adherence, symptom recognition, preventive care pursuance, and lifestyle modification. Beyond the infrastructure of healthcare delivery systems, the service co-production paradigm has many applications. For healthcare research, it empowers an expanded scope of research to readily include patient-centered factors in addition to traditional provider-identified roles. For public health, the paradigm redefines health care system objectives around the patient-centered concept of wellness. 
Table 1 Technobiology examples by discipline

\begin{tabular}{|c|c|c|}
\hline Discipline & Examples & Scope \\
\hline $\begin{array}{l}\text { Biome- } \\
\text { dical }\end{array}$ & $\begin{array}{l}\text { 1. Biological Sensors } \\
\text { 2. Neurostimulator } \\
\text { 3. Hypothermia } \\
\text { 4. Derived Stem Cells } \\
\text { 5. Metrology Tools } \\
\end{array}$ & $\begin{array}{l}\text { 1. Detecting toxic substances } \\
\text { 2. Treating migraine headaches through nerve stimulation, etc. } \\
\text { 3. Lowering body temperature to } 91.5 \text { following cardiac arrest } \\
\text { 4. Employing } 4 \text { embryonic genes to induce stem cell growth } \\
\text { 5. Testing and measuring of nanoscale features }\end{array}$ \\
\hline Chemical & $\begin{array}{l}\text { 1. Regenerative Medicine } \\
\text { 2. DNA Sequencing } \\
\text { 3. Diagnostic Tests } \\
\text { 4. Articoat } \\
\text { 5. Tissue Integrity }\end{array}$ & $\begin{array}{l}\text { 1. Engineering skin, muscle, bone, nerve, bladder, kidney, etc. } \\
\text { 2. Sequencing DNA faster through chips and quantum dots } \\
\text { 3. Testing for Lou Gehrig's, Parkinson's, etc. through genomics } \\
\text { 4. Antimicrobial nanoparticle dressing for wounds } \\
\text { 5. Limiting implant rejection }\end{array}$ \\
\hline Elect & $\begin{array}{l}\text { 1. 3-D Imaging } \\
\text { 2. Robotic } \\
\text { 3. Bioinformatics } \\
\text { 4. Ultrasound } \\
\text { 5. Nanoprobes }\end{array}$ & $\begin{array}{l}\text { 1. Creating 3-D models through multi-photon cell fluorescence } \\
\text { 2. Walking, moving, etc. through automated assist } \\
\text { 3. Analyzing Big Data for drug discovery, etc. } \\
\text { 4. Using focused-ultrasound surgery on fibroid tumors, prostates } \\
\text { 5. Probing temporal changes in cardiac cell potential }\end{array}$ \\
\hline $\begin{array}{l}\text { Environ- } \\
\text { mental }\end{array}$ & $\begin{array}{l}\text { 1. Health Monitors } \\
\text { 2. Hearing Pill } \\
\text { 3. Nano Solar Cells } \\
\text { 4. Protein Characterization } \\
\text { 5. Cell Phenotype }\end{array}$ & $\begin{array}{l}\text { 1. Self-powered sensors that are integrated into fabric. } \\
\text { 2. Preventing hearing loss with naturally available N-acetylcysteine } \\
\text { 3. Developing } 250 \text { nanometer solar film with } 20 \% \text { efficiency } \\
\text { 4. Defining protein structure, function, sequence, concentration, etc. } \\
\text { 5. Defining cell genome, proteome, secretome, metabolism, etc. }\end{array}$ \\
\hline Industrial & $\begin{array}{l}\text { 1. Preventive Care } \\
\text { 2. Adaptive Trials } \\
\text { 3. E-Care } \\
\text { 4. Comprehensive Care } \\
\text { 5. Systems Biology }\end{array}$ & $\begin{array}{l}\text { 1. Predicting through biomarkers/diagnostic tools } \\
\text { 2. Adjusting criteria as clinical trial results are analyzed } \\
\text { 3. Including digital records, wearable wireless devices, etc. } \\
\text { 4. Legislating Patient Protection and Affordable Care Act (PPACA) } \\
\text { 5. Undertaking systematic study of complex biological interactions }\end{array}$ \\
\hline Material & $\begin{array}{l}\text { 1. Nanoparticle Medicine } \\
\text { 2. Drug Delivery } \\
\text { 3. Injectable Bio } \\
\text { 4. Material Biocompatibility } \\
\text { 5. Embedded Transceivers }\end{array}$ & $\begin{array}{l}\text { 1. Targeting special nanoparticles that attach to cancerous cells } \\
\text { 2. Creating drug delivery material with timed release } \\
\text { 3. Using biodegradable elastic polymer to close incisions or cuts } \\
\text { 4. Ensuring that device surface does not cause deleterious effects } \\
\text { 5. Using smart micro-electro-mechanical systems (MEMS) }\end{array}$ \\
\hline $\begin{array}{l}\text { Mecha- } \\
\text { nical }\end{array}$ & $\begin{array}{l}\text { 1. Haptics } \\
\text { 2. Exoskeleton } \\
\text { 3. Body Motion } \\
\text { 4. Artificial Disc } \\
\text { 5. Asthma Mitigation }\end{array}$ & $\begin{array}{l}\text { 1. Sensing/manipulating of objects through touch } \\
\text { 2. Using external anatomical device that supports/protects a body } \\
\text { 3. Harvesting energy for microbial fuel cells } \\
\text { 4. Replacing neck disc, resulting in less pain and swelling } \\
\text { 5. Employing radio-frequency energy to warm airway }\end{array}$ \\
\hline
\end{tabular}

In addition to the technobiology approach of co-production, engineering healthcare must also adopt a systems approach in all its technobiology applications. For example, healthcare must begin to systemically change the way it is organized, paid for, and delivered, including effective diagnostics and therapies (i.e., evidence-based medicine), advances in information and communication technologies (i.e., electronic health records) and new business models (i.e.. 
overcoming the cottage industry structure and the dysfunctional reimbursement and regulatory framework).

The remainder of this paper expands on the technobiology approach of service systems engineering to the development of a healthcare service system that is integrated, adaptive and evidence-driven (Section 2); focuses on a range of example applications in regard to the technobiology areas of information, instrumentation, and insertion (Section 3); highlights, as examples, the collaborative technobiology efforts between the College of Engineering and the Miller School of Medicine at the University of Miami (Section 4); and concludes with some additional insights (Section 5).

\section{Healthcare Service System}

Table 2 Complex service systems: Healthcare system considerations

\begin{tabular}{ll}
\hline System Stages & Healthcare System Considerations \\
\hline 1. Purpose & Stakeholders; Triaging; Business Model \\
2. Boundary & Spatial; Temporal; Interdependent \\
3. Design & Robust; Efficient; Effective \\
4. Development & Models; Scalability; Sustainability \\
5. Deployment & Risk; Uncertainty; Unintended Consequences \\
6. Operation & Flexible; Safe; Secure \\
7. Life Cycle & Predictable; Controllable; Evolutionary \\
\hline
\end{tabular}

As suggested in Table 2, it is difficult, if not impossible, to detail a healthcare service system for several reasons, all of which pertain to the system's complexity. First, the system's purpose is hard to define, given the many stakeholders (i.e., patients, clinicians, insurers, etc.) involved, the multiple objectives (i.e., wellness care, emergency care, acute care, etc.) of each stakeholder, and the overarching business model (i.e., revenues, expenditures, endowments, etc.). How one combines all these divergent viewpoints into a consistent and viable purpose is an almost impossible task. Second, the system's boundary is, at best, ill-defined and shifting; the spatial (i.e., offices, clinics, homes, hospitals, etc.), temporal (i.e., schedules, activities, resources, etc.), and interdependent relationships (i.e., between and among infrastructures, supply chains, demand chains, etc.) are difficult to ascertain. Third, the system's design must be robust (i.e., to insure reliability, quality, integrity, etc.), efficient (i.e., to minimize cost, inventory, waste, etc.), and effective (i.e., to maximize usefulness, satisfaction, pervasiveness, etc.). Fourth, the system's development must be based on models (i.e., gedanken experiments, simulations, networks, etc.), scalability (i.e., multi-scale, multi-level, multi-temporal, etc.), and sustainability (i.e., over time, space, culture, etc.). Fifth, the system's deployment must occur with minimal risk (i.e., morbidity, co-morbidity, mortality, etc.), uncertainty (i.e., unexpected attitude, behavior, performance, etc.), and unintended consequences (i.e., delays, bad side effects, deteriorating vital signs, etc.). Sixth, the system's operation must be flexible (i.e., agile, transparent, redundant, etc.), safe (i.e., with minimal natural accidents, human failures, unforeseen disruptions, etc.), and secure (i.e., with minimal system viruses, system crashes, privacy intrusions, etc.). Seventh, the system's life cycle must be predictable (i.e., in regard 
to inputs, processes, outcomes, etc.), controllable (i.e., with appropriate sensors, feedback, cybernetics, etc.), and evolutionary (i.e., with learning capabilities, timely recoveries, intelligent growth, etc.).

Nevertheless, we can consider healthcare to be the treatment and management of illness, and the preservation of health through services offered by the medical, dental, pharmaceutical, clinical, nursing, and allied health professions. Healthcare embraces all the goods and services designed to promote health, including "preventive, curative and palliative interventions, whether directed to individuals or to populations" ${ }^{[2]}$. As detailed in our earlier papers ${ }^{[3]}$ and briefly summarized herein for convenience, healthcare services should be carried out with knowledge-intensive agents or components who work together as providers and consumers to create or co-produce value. Indeed, the engineering design of a healthcare system must recognize the fact that it is actually a complex integration of human-centered activities that is increasingly dependent on information technology and knowledge. Like any service system, healthcare can be considered to be a combination or recombination of three essential components - People (characterized by behaviors, values, knowledge, etc.), processes (characterized by collaboration, customization, etc.) and products (characterized by devices, software, etc.). Thus, a healthcare system is an integrated, adaptive and evidence-driven set of people, processes and products. It is, in essence, a system of systems which objectives are to enhance its efficiency (leading to greater interdependency) and effectiveness (leading to improved health).

\section{$2.1 \quad$ Integrated}

Unfortunately, in spite of the provisions included in Public Law 111-148, the major healthcare reform bill signed by President Obama on March 23, 2010, the US healthcare remains in disarray - Essentially, a non-system. The US healthcare is a good example of a peopleintensive, expensive and, yet, among the least effective system for a developed country; a minority of the population receives excellent care, while an equal minority receives inadequate care $^{[4]}$. This situation is not due to a lack of well-trained health professionals or to a lack of innovative technologies; it is due to the fact that it is based on a fragmented group of mostly small, independent providers driven by cost-obsessed insurance companies ${ }^{[5]}$. As a consequence, an integrated healthcare system must be developed and implemented, one requiring the participation and support of a large number of stakeholders (i.e., consumers, doctors, hospitals, insurance companies, etc.). For example, patients must take increased responsibility for their own healthcare in terms of access to and use of validated information. In sum, U.S. healthcare must become a co-produced, technobiology-oriented system.

Moreover, by employing the integrative approach of systems engineering, the US healthcare imbalance can be better equilibrated, resulting in a more efficient and effective system. In this regard, payment for care should be more weighted to output or value provided than to input or activities undertaken. Additionally, such production techniques as cross-training and multi-use of facilities can enhance both efficiency and effectiveness. All of these issues must be taken into consideration as President Obama and Congress seek to improve US healthcare; public plans (e.g., Medicare and Medicaid) must be integrated with a plethora of private plans (that are, unfortunately, neither affordable nor portable) in order to achieve healthcare coverage for all US residents. 
As mentioned earlier, a service system like healthcare is actually an integration or combination of three essential components - People, processes and products. In particular, people can be grouped into those demanding services (i.e., consumers, users, patients, buyers, organizations, etc.) and those supplying the services (i.e., suppliers, providers, clinicians, servers, sellers, organizations, etc.); although sometimes ad hoc, processes are primarily procedural (i.e., standardized, evolving, decision-focused, network-oriented, etc.) and/or algorithmic (i.e., data mining, decision modeling, systems engineering, etc.) in structure; and products can be physical (i.e., facilities, sensors, information technologies, etc.) or virtual (i.e., e-commerce, simulations, e-collaboration, etc.) in form.

Given the co-producing nature of services, it is obvious that people constitute the most critical component or element of a service system. However, because people are so unpredictable in their values, behaviors, attitudes, expectations, and knowledge, they invariably raise the complexity of a service system. Moreover, the multi-stakeholder - and related multi-objective nature of such systems serve to only intensify the complexity level and may render the system to be undefinable, if not unmanageable. Human performance, social networks and interpersonal interactions combine to further aggravate the situation. Other barriers to co-production are considered by Parasher et al. ${ }^{[6]}$. Processes that underpin system integration include standards, procedures, protocols, and algorithms. By combining or integrating service processes, one could, for example, establish a "one-stop shopping" approach, a highly desirable situation for the consumer or customer. However, integration of financial services has resulted in giant banks (e.g., Citigroup); integration of home building goods and services has resulted in super box stores (e.g., Home Depot); and integration of software services has resulted in complex software packages (e.g., Microsoft Office). Integration also enhances system efficiency, if not its effectiveness. For example, the radio frequency location and identification (RFLID) tag or computer chip with a transmitter - serves to integrate the supply chain. Unfortunately, as supply chains become more electronically integrated and interdependent, cyber security becomes more problematic.

As indicated in Table 3, service system integration can occur over the physical, temporal, organizational and functional dimensions. Physical integration can be defined by the degree of systems co-location in the natural (i.e., closed, open, hybrid), constructed (i.e., goods, structure, systems) or virtual (i.e., service, simulated, e-commerce) environment. An urban center's infrastructures (e.g., emergency services, health services, financial services, etc.) are examples of a constructed environment. Over time and with advances in information technology and the necessity for improved efficiency and effectiveness, these infrastructures have become increasingly automated and interlinked or interdependent. In fact, because the information technology revolution has changed the way business is transacted, government is operated, and national defense is conducted, the US President ${ }^{[7]}$ singled it out as the most critical infrastructure to protect following the disaster of $9 / 11$. Thus, while the US is considered a superpower because of its military strength and economic prowess, non-traditional attacks on its interdependent and cyber-underpinned infrastructures could significantly harm both the nation's military power and economy, including that of the healthcare sector. Clearly, infrastructures, especially the information infrastructure, are among the nation's weakest links; they are vulnerable to willful 
acts of sabotage, if not invasions of privacy. Moreover, this interdependency contributed significantly to the 2008 economic disaster or recession. Recent efforts at imbuing infrastructures with "intelligence" make it increasingly feasible to address the safety and security concerns, allowing for the continuous monitoring and real-time control of critical infrastructures.

Temporal integration can be defined by the degree of systems co-timing from a strategic (i.e., analytical, procedural, political), tactical (i.e., simulation, distribution, allocation), and operational (i.e., cognition, visualization, expectation) perspective. Expectation, for example, is a critical temporal issue in the delivery of services. More specifically, since services are to a large extent subject to customer satisfaction and since, as Tien and Cahn ${ }^{[8]}$ postulated and validated, "satisfaction is a function of expectation," service performance or satisfaction can be enhanced through the effective "management" of expectation. When applied to healthcare, however, it may be difficult, if not impossible, to manage a patient's expectation under certain emergency situations.

Organizational integration can be defined by the degree of systems co-management of resources (i.e., people, processes, products), economics (i.e., supply, demand, revenue), and management (i.e., centralized, decentralized, distributed). In regard to management integration, Tien et al. ${ }^{[9]}$ provide a consistent approach to considering the management of both goods and services - By first defining a value chain and then showing how it can be partitioned into supply and demand chains, which, in turn, can be appropriately managed. Of course, the key purpose for the management of supply and demand chains is to smooth-out the peaks and valleys commonly seen in many supply and demand patterns, respectively. Moreover, real-time mass customization occurs when both supply and demand chains are simultaneously managed. The shift in focus from mass production to mass customization (whereby a service is produced and delivered in response to a customer's stated or imputed needs) is intended to provide superior value to customers by meeting their unique needs. It is in this area of customization where customer involvement is not only at the goods design stage but also at the manufacturing or co-production stage - that services and manufacturing are merging in concept, resulting in a "servgood" (which is further defined in Section 3).

Functional integration can be defined by the degree of systems co-functioning in regard to input (i.e., location, allocation, re-allocation), process (i.e., informatics, feedback, control), and output (i.e., efficiency, effectiveness). From an output perspective, for example, it is obvious that a system should be about integrating and enhancing efficiency and effectiveness, the twin pillars of productivity. However, it should be noted that efficiency is mostly related to the manner in which the supply chain has been designed for optimal operation, while effectiveness is mostly related to the manner in which the demand chain has been responsive to consumer needs.

Again, healthcare - as a service system - must be integrated in regard to people, processes and products, as well as over the physical, temporal, organizational and functional dimensions. It is obvious that designing an efficient and effective healthcare system is not easily accomplished; socialistic systems like Sweden's cost too much, while capitalistic systems like the US's are both high cost and not fair. New design approaches are required. The information technology revolution has permitted the analysis part of system design to be largely undertaken 
by computers; it allows for a simulated and collaborative redesign process to occur - until a satisfactory design is achieved which meets specified performance (e.g., morbidity, mortality, cost, etc.) criteria. The resultant and integrated healthcare system must be a comprehensive, interoperable system of systems. Perhaps the best example of an integrated healthcare system is that proposed by Goldschmidt-Clermont et al. ${ }^{[10]}$; based on the autonomic computing initiative, they propose an autonomic healthcare system that combines the existing hospital information technology with operational processes to bring down the barriers among different specialties and to improve the quality of care being provided. As a final point, it should be noted that the human body is itself an amazingly integrated system of systems, one that should not, if possible, be perturbed by surgeries and other intrusive treatments; perhaps the clinical specialist that should command the highest salary is one who focuses on the body as a system of systems.

\subsection{Adaptive}

Because a service system is, by definition, a co-producing system, it must be adaptive. Adaptation is a uniquely human characteristic, based on the technobiology approach of decision making. (Indeed, designing a healthcare system is about making decisions or choices about the system's characteristics or attributes.) Tien ${ }^{[11]}$ underscores the difference between data and information, especially from a decision making perspective. Data represent basic transactions captured during operations, while information represents processed data (e.g., derivations, groupings, patterns, etc.). Clearly, except for simple operational decisions, decision making at the tactical or higher levels requires, at a minimum, appropriate information or processed data; moreover, electronic data technology has transformed large-scale information systems from being the "glue" that holds the various units of an organization together to being the strategic asset that provides the organization with its competitive advantage.

The fact remains that data - both quantitative and qualitative - need to be effectively and efficiently fused and analyzed in order to yield appropriate information for informed or intelligent decision making in regard to the design, production and delivery of goods and services, including healthcare. At present, decision modeling methods suffer from two shortcomings. First, most of the available - especially optimization - methods are only applicable in a steady state environment, whereas in the real-world, all systems are in transition. (Note that steady-state, like average, is an analytical concept that allows for a tractable, if not manageable, analysis.) Second, most of the available methods are unable to cope with changing circumstances; instead, we need methods that are adaptive so that decisions can be made in real-time, as is required in most healthcare situations. Thus, non-steady-state, adaptive decision methods are required. More importantly, real-time decision modeling is not just about speeding up the models and solution algorithms; it, like real-time data fusion and analysis, also requires additional research and development.

Again, the real-time nature of co-producing services - especially human-centered services that are computationally-intensive and intelligence-oriented - requires a real-time, systems engineering approach. Ethnography, a branch of anthropology that can help to identify a consumer's unmet needs, is being used to spot breakthrough product and service innovations. Another critical aspect of systems engineering is system performance; it provides an essential 
framework for assessing the decisions made - in terms of such issues as satisfaction, convenience, privacy, security, equity, quality, productivity, safety and reliability. Similarly, undertaking systems engineering within a real-time environment will require additional thought and research.

Human interface is another essential element of an adaptive service system; it is actually a critical tool in systems engineering. Such interface could include the interactions between and among humans and software agents, machines, sub-systems, and systems of systems. Human factors constitute a discipline that deals with many of these interactions. However, another critical interface concerns how humans interact with data and information. In developing appropriate human-information interfaces, one must pay careful attention to a number of factors. First, human-information interfaces are actually a part of any decision support model; they structure the manner in which the model output or information is provided to the decision maker. Cognition represents the point of interface between the human and the information presented. The presentation must enhance the cognitive process of mental visualization, capable of creating images from complex multidimensional data, including structured and unstructured text documents, measurements, images and video. Second, constructing and communicating a mental image common to a team of, say, clinicians and nurses could facilitate collaboration and could lead to more effective decision making at all levels, from operational to tactical to strategic. Nevertheless, cognitive facilitation is especially necessary in operational settings which are under high stress. Third, cognitive modeling and decision making must combine machine learning technology with a priori knowledge in a probabilistic data mining framework to develop models of, say, a nurse's tasks, goals, and objectives. These user-behavior models must be designed to adapt to the individual decision maker so as to promote better understanding of the needs and actions of the individual, including adversarial behaviors and intents.

More importantly and as detailed in Table 4, service system adaptation can occur over the monitoring, feedback, cybernetic and learning dimensions. Monitoring adaptation can be defined by the degree of sensed actions in regard to data collection (i.e., sensors, agents, swarms), data analysis (i.e., structuring, processing, mining), and information abstraction (i.e., derivations, groupings, patterns). Data are acquired by sensors, which could be in the form of humans, robotic networks, aerial images, radio frequency signals, and other measures and signatures. In regard to patients, for example, sensors which monitor their vital signs are essential, as are verbal inputs from the patients themselves. More recently, data warehouses residing in the cloud are proliferating and Big Data mining techniques are gaining in popularity. However, no matter how large a data warehouse and how sophisticated a data mining technique, problems can occur if the provided data do not possess the desired attributes of measurability, availability, consistency, validity, reliability, stability, accuracy, independence, robustness, completeness and timeliness.

Feedback adaptation can be defined by the degree of expected actions based on standardized (i.e., pre-structured, pre-planned), procedural (i.e., policies, standard operating procedures), and algorithmic (i.e., optimized, Bayesian) approaches. In general, models underpin these approaches. As an example, Kaplan et al. ${ }^{[12]}$ have developed a set of complex models to demonstrate that the best prevention approach to a smallpox attack would be to undertake 
immediate and widespread vaccination. Unfortunately, models, including simulations, dealing with multiple systems are still relatively immature and must be the focus of additional research and development. Such system of systems models are quite complex - especially in regard to a possible misalignment of the underlying system objectives - and will require a multidisciplinary approach.

Table 3 System integration: Dimensions

\begin{tabular}{llll}
\hline Dimension & Definition & Characteristics & Elements \\
\hline \multirow{2}{*}{ Physical } & Degree of Systems & Natural & Closed; Open; Hybrid \\
& Co-Location & Constructed & Goods; Structures; Systems \\
& & Virtual & Services; Simulation; E-Commerce \\
\hline \multirow{2}{*}{ Temporal } & Degree of Systems & Strategic & Analytical; Procedural; Political \\
& Co-Timing & Tactical & Simulation; Distribution; Allocation \\
& & Operational & Cognition; Visualization; Expectation \\
\hline \multirow{3}{*}{ Organizational } & Degree of Systems & Resources & People; Processes; Products \\
& Co-Management & Economics & Supply; Demand; Revenue \\
& & Management & Centralized; Decentralized; Distributed \\
\hline \multirow{2}{*}{ Functional } & Degree of Systems & Input & Location; Allocation; Re-Allocation \\
& Co-Functioning & Process & Informatics; Feedback; Control \\
& & Output & Efficiency; Effectiveness \\
\hline
\end{tabular}

Table 4 System adaptation: Dimensions

\begin{tabular}{llll}
\hline \multirow{2}{*}{ Dimension } & Definition & Characteristics Elements \\
\hline \multirow{2}{*}{ Monitoring } & Degree of Sensed & Data Collection & Sensors; Agents; Swarms \\
& Actions & $\begin{array}{l}\text { Data Analysis } \\
\text { Information }\end{array}$ & Structuring; Processing; Mining \\
& & Abstraction & \\
\hline \multirow{2}{*}{ Feedback } & Degree of Expected & Standardized & Pre-Structured; Pre-Planned \\
& Actions & Procedural & Policies; Standard Operating Procedures \\
& & Algorithmic & Optimized; Bayesian \\
\hline \multirow{2}{*}{ Cybernetic } & Degree of Reactive & Deterministic & Known States; Deterministic Actions \\
& Actions & Dynamic & Known State Distributions; Dynamic Actions \\
& & Adaptive & Unknown States; Adaptive Actions \\
\hline \multirow{2}{*}{ Learning } & Degree of Unstruc- & Cognition & Recognition-Based; Behavioral \\
& tured Actions & Evidence & Information-Based; Genetic \\
& & Improvisation & Experience-Based; Evolutionary \\
\hline
\end{tabular}

Cybernetic adaptation can be defined by the degree of reactive actions that could be deterministic (i.e., known states, deterministic actions), dynamic (i.e., known state distributions, dynamic actions), or adaptive (i.e., unknown states, adaptive actions). Cybernetics is derived 
from the Greek word "kybernetics", which refers to a steersman or governor. Within a system, cybernetics is about feedback (through evaluation of performance relative to stated objectives) and control (through communication, self-regulation, adaptation, optimization, and/or management); thus, cybernetic adaptation refers to actions that are undertaken based on an assessment of the feedback signals and then taking corrective steps to control the system so as to achieve the desired system objectives. As an example, autopilots - which are programmed to deal with deterministic and dynamic situations - can, for the most part, take off, fly and land a plane; yet, usually two human pilots are also on the plane, just in case an unknown state occurs and the adaptive judgment of a human pilot is required. Clearly, a trained human - like a clinician or surgeon - is still the most adaptive controller, although machines are becoming more 'intelligent' through adaptive learning algorithms.

System control is perhaps the most critical challenge facing designers of multiple, interrelated systems or system of systems (SoS). Due to the difficulty, if not impossibility, of developing a comprehensive SoS model, either analytically or through simulation, SoS control remains an open problem and is, of course, uniquely challenging for each application domain, especially in regard to healthcare. Moreover, real-time control - which is required in almost all application domains - of interdependent systems poses an especially difficult problem. The cooperative control of a SoS assumes that it can be characterized by a set of interconnected systems or agents with a common goal, which unfortunately is not the case with most healthcare systems. Classical techniques of control, optimization and estimation could be used to create parallel architectures for, as an example, coordinating numerous sensors. However, many issues dealing with real-time cooperative control have not been addressed, even in non-SoS structures. For example, one issue concerns the control of a SoS in the presence of communication delays among the SoS sub-systems.

Finally, learning adaptation can be defined by the degree of unstructured actions based on cognition (i.e., recognition-based, behavioral), evidence (i.e., information-based, genetic), and improvisation (i.e., experience-based, evolutionary). Learning adaptation is mostly about real-time decision making at the operational level. In such a situation and as indicated earlier, it is not just about speeding up steady-state models and their solution algorithms; in fact, steady-state models become mostly irrelevant in real-time environments. Instead, it concerns reasoning under both uncertainty and severe time constraints. The rise of cloud computing whereby the vast array of servers (including laptops and smartphones) can be connected to data and algorithms almost anytime and anywhere - is becoming an essential tool to computational decision making, including improvisation.

At all levels of healthcare decision making, there are a spectrum of possible methods that can be utilized, ranging from adaptive - instead of randomized - medical trials, to autonomous control, to virtual-touch tools, to genetic algorithms, to improvisation, all able to cope with imprecision, uncertainties and partial truth. As an example, shared or informed decision making is becoming more popular and, as a result, patients are electing to have fewer surgeries for clogged arteries (when they are informed that, except for reducing chest pains, drugs are just as effective as angioplasty surgeries - with balloons and stents - in preventing heart attacks and death), prostate cancer (when they are informed that 97 percent of men with prostate cancer 
die of some other cause), and herniated discs (when they are informed that the outcomes are the same whether they have surgery or not). Moreover, the methods can be used to process information, take into account changing conditions, and learn from the environment; thus, they are adaptive and, to a large extent, responsive to a data stream of real-time input. In a fully integrated and adaptive SoS, each system must be able to communicate and interact with the entire SoS, without experiencing compatibility issues.

\subsection{Evidence-Driven}

The venue for evidence is increasingly connected with the emergence of e(lectronic)-services; they include, as examples, medical records, financial services, banking, airline reservation systems, and consumer goods marketing. As discussed by Tien and Berg ${ }^{[13]}$, e-service enterprises interact or "co-produce" with their customers in a digital (including e-mail and Internet) medium, as compared to the physical environment in which traditional or bricks-and-mortar service enterprises interact with their customers. Similarly, in contrast to traditional services which include low-wage "hamburger flippers", e-services typically employ high-wage earners and services that are more demanding in their requirements for self-service, transaction speed, and computation. In regard to data input that could be processed to produce information that, in turn, could be used to help make informed service decisions, it should be noted that both sets of services rely on multiple data sources; however, traditional services typically require homogeneous (mostly quantitative) data input, while e-services increasingly require non-homogeneous (i.e., both quantitative and qualitative) data input. Paradoxically, the traditional service enterprises have been driven by data, although data availability and accuracy have been limited (especially before the pervasive use of the Universal Product Code - UPC - and the more recent deployment of radio frequency location and identification - RFLID - tags).

Consequently, while traditional services are based on economies of scale and a standardized approach, e-services emphasize economies of expertise or knowledge and an adaptive approach. Another critical distinction between traditional and e-services is that, although all services require decisions to be made, traditional services are typically based on predetermined decision rules, while e-services require real-time, adaptive decision making; that is why Tien ${ }^{[11]}$ has advanced a decision informatics paradigm, one that relies on both information and decision technologies, in real-time. High-speed Internet access, cloud computing, wireless networks, electronic sensors and ever-smarter software are the tools for building a global services economy. Thus, in e-commerce, a sophisticated and integrated service system combines product (i.e., good and/or service) selection, order taking, payment processing, order fulfillment and delivery scheduling into a seamless system, all provided by distinct service providers; in this regard, it can be considered to be a system of different systems.

Although the comparison between services and physical goods highlights some obvious methodological differences, it is interesting to note that the physical goods depreciate with use and time, while the virtual service assets are generally reusable and may in fact increase in value with repeated use and over time. The latter assets are predominantly processes and associated human resources that build on the skill and knowledge base accumulated by repeated interactions with the service receiver, who is involved in the co-production of the service. Thus, for example, a surgeon should get better over time, especially if the surgical procedure is re- 
peated. Indeed, clinical productivity increases for an average physician, from the dawn of a career to almost the end of a career, with a slight slowing down towards the end. Likewise, while most US physicians practice at a financial loss over the first few years, they progressively improve their financial standing over the course of their career.

Another critical influence on services is the computationally-driven move towards mass customization. "Customization" implies meeting the needs of a customer market that is partitioned into an appropriate number of segments, each with similar needs (e.g., Amazon.com targets their marketing of a new book to an entire market segment if several members of the segment act to acquire the book). "Mass customization" implies meeting the needs of a segmented customer market, with each segment being a single individual (e.g., a tailor who laser scans an individual's upper torso and then delivers a uniquely fitted jacket). And "real-time mass customization" implies meeting the needs of an individualized customer market on a real-time basis (e.g., a tailor who laser scans an individual's upper torso and then delivers a uniquely fitted jacket within a reasonable period, while the individual is waiting). To a large extent, one could argue that healthcare is, almost by definition, a real-time mass customized service; however, at the present time, healthcare is not computationally-driven and therefore not cost-effective. Nevertheless, it is interesting to note that in regard to customization and in relation to the late 1700s, the U.S. is in some respects going "back-to-the-future"; thus, advanced technologies are not only empowering the individual but are also allowing for individualized or customized goods and services. Moreover, when mass customization occurs, it is difficult to say whether a service or a good is being delivered; that is, an expert-guided robotic surgery - like a uniquely fitted jacket - can be considered to be a co-produced service and good or, as defined in Section 3 , "servgood".

Clearly, healthcare needs to transition from being a traditional (although high-wage) service to an electronic-based service industry, one relying on digital media for such activities as realtime access to patient records and consultation at-a-distance. Additionally, healthcare must adopt some of the methods that have enabled manufacturing to be efficient (e.g., reduced cycle time, improved quality, etc.), while focusing on service effectiveness (e.g., maintaining a high standard of co-production, meeting patient expectation, etc.). Most importantly, healthcare must be adaptive and must customize their treatments to the needs of their patients, ranging from evidence-based protocols to personalized medications.

\section{Example Applications}

Before discussing a range of example technobiology applications, three remarks should be made. First, according to economists, a nation's economy is divided into two major sectors: the services sector includes a number of large industries (i.e., wholesale, retail, business, education, government, healthcare, etc.) that employs - in the US - over 80 percent of the workers, and the goods sector (i.e., manufacturing, construction, agriculture, and mining) that employs the remaining - less than 20 percent of - workers. In practice, the delineation between the different economic sectors is quite blurred; this is especially true between the manufacturing and service sectors, which are highly interdependent ${ }^{[13]}$. The interdependences, similarities and complementarities of services and goods are significant. Indeed, many of the manufacturing 
innovations are relevant to the services industries. It is critical that the co-producing nature of services be carefully taken into consideration. For example, in manufacturing, physical parameters and statistics of production and quality can be more precisely identified; in contrast, since a services operation depends on an interaction between the process of producing the service and the recipient, the characterization is, as noted earlier, necessarily more subjective or expectation-related ${ }^{[8]}$. Moreover, in services, automation-driven software algorithms have transformed human resource-laden, co-producing services systems to software algorithm-laden, self-producing services. Thus, extensive manpower would be required to manually co-produce the services if automation were not available. Although automation has certainly improved productivity and decreased costs in some services (e.g., telecommunications, internet commerce, etc.), it has not yet had a similar impact on other labor-intensive services (e.g., healthcare, education, etc.). Nevertheless, with new multimedia and broadband technologies, some hospitals are personalizing their treatment of patients, including the sharing of electronic records with their patients.

What then is a servgood and, more specifically, a connected servgood? Figure 1 provides a definition in terms of an illustration. A servgood can be thought of as a physical good layer enveloped by a services-oriented layer that makes the good smarter or more adaptable and customizable for a particular use. Another layer of physical sensors (e.g., actuators, radio frequency identification tags, global positioning systems, etc.) could then enhance its smartness and adaptability, especially if it were to be connected to similar or different servgoods, usually through the Internet of Things. A sensor is obviously a good or a device that detects events or changes in events and provides a corresponding output, generally as an electrical or optical signal. Sensors also serve to enhance an individual's five traditional senses of sight, hearing, taste, smell, and touch. Since sensors are active devices, they must be powered, hopefully by inexpensive and small batteries; interestingly, as sensors become smaller, some are able to be powered by battery-free ambient motions or even radio frequency backscatter from cellular and television transmissions. Actually, Figure 1 depicts an internet of connected servgoods ${ }^{[14]}$; that is, if the "good" were to represent a basic car and the "service" were to encompass electronic assists, then the "servgood" would be a smart car that together with sensor access and connectivity to the Internet could be become a member of a community of smart driverless (i.e., autonomous) cars! In sum, the goods layer is customized through the services layer and is then connected through the sensors layer to the Internet via, say, WiFi, WiMax or NFC (near field communications). Servgoods, especially connected servgoods, are now the focus of a growing number of innovative enterprises - certainly, the Apple iPhone can be considered to be among the very first connected servgood. Other smart, cyber-enabled servgoods include the electric grid, transportation, buildings, medical devices, brain-mimicked microchips, drones, wearables, cities, health, homes, shopping, uber - all considered to be smart, connected products that "combine hardware, sensors, data storage, microprocessors, software and connectivity in myriad ways" ${ }^{[15]}$. Indeed, in a recent issue, the influential MIT Technology Review ${ }^{[16]}$ listed the top 50 smartest (defined as those which "made strides in the past year that will define its field") companies in 2014; interestingly, as indicated in Table 5, six (i.e., Tesla, Google, Samsung, Dropbox, BMW and Amazon) or 60 percent of the list's top 10 companies have products that 
can be considered to be connected servgoods! It should be noted that, unlike the iPhone, many of the more complex connected servgoods are not yet ready for prime time; for example, it is estimated that while cars will talk among themselves in 5 years, it will take an additional 5 years for them to collaborate through embedded cognition.

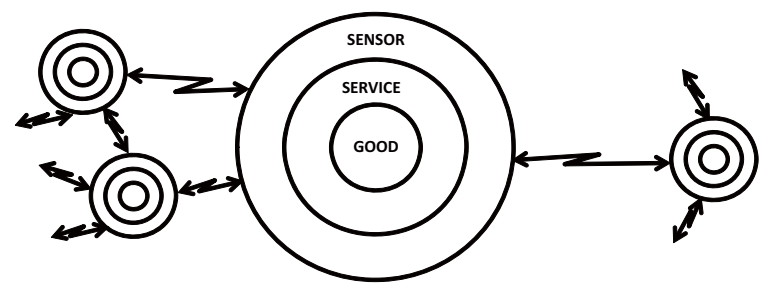

Figure 1 Internet of sensed servgoods

Table 5 Top 10 smartest companies in 2014 (MIT Technology Review)

\begin{tabular}{llllll}
\hline Focus & Scope & Goods & Services & Servgoods & $\begin{array}{l}\text { Connected } \\
\text { Servgoods }\end{array}$ \\
\hline 1. Illumina & Genome sequencing & - & $\mathrm{X}$ & - & - \\
2. Tesla & Electric cars & $\mathrm{X}$ & $\mathrm{X}$ & $\mathrm{X}$ & $\mathrm{X}$ \\
3. Google & Android, navigation, etc. $\mathrm{X}$ & $\mathrm{X}$ & $\mathrm{X}$ & $\mathrm{X}$ \\
4. Samsung & Smartphone & $\mathrm{X}$ & $\mathrm{X}$ & $\mathrm{X}$ & $\mathrm{X}$ \\
5. Salesforce & Incorporate IoT data & - & $\mathrm{X}$ & - & - \\
6. Dropbox & Cloud file storage & $\mathrm{X}$ & $\mathrm{X}$ & $\mathrm{X}$ & $\mathrm{X}$ \\
7. BMW & Self-driving capabilities & $\mathrm{X}$ & $\mathrm{X}$ & $\mathrm{X}$ & $\mathrm{X}$ \\
8. ThirdRock & Biotech ventures & - & $\mathrm{X}$ & - & - \\
9. Square & Email payment systems & - & $\mathrm{X}$ & - & - \\
10. Amazon & E-commerce & $\mathrm{X}$ & $\mathrm{X}$ & $\mathrm{X}$ & $\mathrm{X}$ \\
\hline
\end{tabular}

\subsection{Information}

Clearly, with all of the data being generated by smart sensors or connected servgoods, there are yottabytes $\left(1000^{* * 8} 8\right.$ bits) of data available, even just in the medical or healthcare domain. Such Big Data - A term applied to data sets whose size is beyond the ability of available tools to undertake their acquisition, access, analytics and/or application in a reasonable amount of time - must be transformed into useful information. Whereas Tien ${ }^{[11]}$ forewarned about the data rich, information poor (DRIP) problems that have been pervasive since the advent of large-scale data collections or warehouses, the DRIP conundrum has been somewhat mitigated by the Big Data approach which has unleashed information in a manner that can support informed - yet, not necessarily defensible or valid - decisions or choices. Big Data is poised to add greater value to businesses (which can fathom their own transactional data to detect patterns suggesting the effectiveness of their pricing, marketing and supply chain strategies); to understand planet earth (which is being extensively monitored on the ground, in the air and in the oceans); to solve science and engineering problems (which have become more data- or empirically-driven); to enhance the World Wide Web (which is amassing terabytes of textual, audio and visual material that is becoming widely available through search engines like Google, Yahoo and Bing); to aid national security agencies (which are collecting and mining satellite and thermal imagery, audio intercepts and other readily available data and information); and, 
more specific to our current focus, to support modern medicine (which is collecting and mining large amounts of image scans and genetic markers). In a recent special issue of the Spectrum, IEEE $^{[17]}$ employs Big Data to hack the human operating system code (similar to what medicine is attempting to do in understanding the human body); a series of articles reports on first reading the code, then analyzing the code, and finally attempting to change the code through microbots, cyborgs, Watsons and brain hacking.

Table 6 Big Data concerns

\begin{tabular}{lll}
\hline Components & Elements & Potential Concerns \\
\hline Acquisition & Focus & Big data does not imply big/complete understanding of underlying problem \\
& Emphasis & Big data quantity does not imply big data quality \\
& Scope & Big data sample does not imply a representative or even a complete sample \\
\hline Access & Focus & Big data's on-demand accessibility may create privacy or big brother concerns \\
& Emphasis & Big data's real-time abilities may obscure past and future concerns \\
& Scope & Big data's cyber-security concerns may overlook personal-security concerns \\
\hline \multirow{2}{*}{ Analytics } & Focus & Big data's inherent messiness may obscure underlying statistical relationships \\
& Emphasis & Big data's correlational finding may result in an unintended causal consequence \\
& Scope & Big data's unleashing of information may obscure underlying truths \\
\hline Application & Focus & Big data's feasible explanations may obscure more probable explanations \\
& Emphasis & Big data's evidence-driven findings may obscure underlying factual knowledge \\
& Scope & Big data's subjective, consumer-centric findings may obscure simpler objective \\
& findings \\
\hline
\end{tabular}

While Big Data allows for a) better integration or fusion and subsequent analysis of quantitative and qualitative data, b) better observation of rare but great impact events or "black swans" ${ }^{[18]}$, c) greater system and system-of-systems efficiency and effectiveness; d) better evidence-based - "data rich, information unleashed" (DRIU) - decisions that can overcome the prejudices of the unconscious mind, and e) more messy findings but yet good enough to support informed decisions, there are still some critical concerns ${ }^{[19]}$. Table 6 provides a summary of the concerns, in terms of the four data processing components: acquisition (i.e., Big Data does not imply big/complete understanding of underlying problem; Big Data quantity does not imply Big Data quality; and Big Data sample does not imply a representative or even a complete sample); access (i.e., Big Data's on-demand accessibility may create privacy or Big Brother concerns; Big Data's real-time abilities may obscure past and future concerns; and Big Data's cyber-security concerns may overlook personal-security concerns); analytics (i.e., Big Data's inherent messiness may obscure underlying relationships; Big Data's correlational findings may result in unintended causal consequences; and Big Data's unleashing of information may obscure underlying truths); and application (i.e., Big Data's feasible explanations may obscure more probable explanations; Big Data's evidence-driven findings may obscure underlying factual knowledge; and Big Data's subjective, consumer-centric findings may obscure simpler objective findings).

Other concerns include surveillance by autocratic governments and processing data in an increasingly unfocused, unproductive and generally "shallow manner" ${ }^{[20]}$. Moreover, even 
Google's vaunted flu prediction algorithm (which in 2009 was able to predict and locate the H1N1 flu spread on a near real-time basis) failed in 2012; it was predicting more than double the proportion of doctor visits for influenza-like illnesses than the Centers for Disease Control and Prevention (which bases its estimates on a survey of clinics located throughout the US) - underscoring the fact that correlation does not necessarily imply causation. Of course, the potential Big Data concerns or problems can be mitigated with thoughtful and effective approaches and practices; for example, legislation could be promulgated and passed to forbid the invasion of privacy and to dispense severe sanctions against those who break the law or who knowingly publish false findings. Alternatively, a watch dog organization can be created to discover such findings, much like the recently established METRICS (Meta-Research Innovation Centre at Stanford) which mission is "identifying and minimizing persistent threats to medical research quality".

\subsection{Instrumentation}

More recently, much of the focus on instrumentation in the healthcare area is on wearables, including smart phones and devices, self-powered sensors in clothing, and even tattoos printed on the body, all to monitor vital signs - including blood pressure, glucose, body temperature, respiratory rate, airborne pollutants, and ambient temperature. (Parenthetically, it should be noted that some of wearables may cause interference; for example, some tattoos can block light from a smart watch sensor.) Interestingly, the psychology behind wearables is that the obtained data would help the wearers to achieve, if not exceed, some daily goal of fitness. However, according to market researchers, while at least 1 in 10 Americans own a tracking device like Fitbit and Nike Fuelband, more than a third of those who possess such a gadget abandon them within a few months. The reasons for abandonment include loss of interest, discomfort, and meaningless output (e.g., after a few days, the wearer has a good feel of when the daily goal is met and/or exceeded).

More sophisticated sensors are forthcoming. Retinal imaging and the ability to view the underlying blood vessels can reveal the presence of several disorders, including heart disease, strokes, diabetes, multiple sclerosis, and Alzheimer's. In the near future, retina selfies may help physicians detect such disorders in a timely manner so that appropriate preventative treatments can be successfully prescribed.

Experimental psychologists are now studying ways of motivating individuals beyond the feedback of data from wearables; instead, they must be motivated on a subconscious level to take charge of their health. Actually, the popularity of wearable trackers in the recent past has been partly due to outside incentives, including insurers providing discounts on premiums if vital signs improve, gyms installing televisions in front of treadmills, instructor-facilitated exercises (e.g., Pilates), game-directed exercises (e.g., Zombies, Run!), and social media-reinforced goal setting. In sum, successful wearables should support people making commitments to themselves and others.

\subsection{Insertion}

More and more of the sensors, medicines and replacement parts that are inserted into the body are being made not only with new and compatible materials but also employing a 
technology-driven, layer-by-layer assembly of thin films, sometimes referred to as 3-D Printing. In this manner, one can have nanometer control of each film's material and thickness for a range of applications, including membranes, catalysis, biomedicine, and optics. The assembly technologies - including dipping, de-wetting, centrifugation, spinning, spraying, atomization, electrodeposition, electro-coupling, microfluidics, and filtration - can also have an impact on the performance and physicochemical properties of the nanofilms. Scaling up layer-by-layer assembly or printing is crucial for carrying out real-world applications. Also, the assembly technologies must be compared and assessed to gauge their ability to coat particulate substrates.

\section{UM Experience}

Here, at the University of Miami (UM), the Miller School of Medicine (MSoM) and the College of Engineering $(\mathrm{CoE})$ are collaborating in a range of areas that have underscored the importance of innovations in technobiology (whereby biology is enhanced by appropriately applying our understanding of technology), as well as in biotechnology (whereby technology is enhanced by appropriately applying our understanding of biology). While we, the authors and deans of our respective academic units, began our collaboration in 2007, it should be noted that there were several instances of successful collaboration among our faculties before we arrived on the scene; however, most would agree that we formalized, if not significantly enhanced, the level of collaboration between our two units.

As professionals, we began our collaboration with a number of constructive discussions which established the fact that we were like-minded in a number of ways, leading eventually to several jointly authored publications ${ }^{[6,21]}$. We formally introduced the technobiology - versus the overused biotechnology - concept on February 25, 2010, when we convened a national panel of nine renowned engineering and medical experts, all members of the US National Academies of Engineering and Medicine (formerly known as the Institute of Medicine), to discuss the impact of technology on improving healthcare for the benefit of humanity. The well-attended regional meeting on "Engineering Innovations in Healthcare" was a co-sponsored event, a recent first for the two National Academies and it featured three organizational leaders - the two National Academy Presidents and the President of the University of Miami. The panel unanimously agreed that technology can mitigate, if not solve, many of the pressing issues in healthcare and biology, ranging from making Obamacare work to developing effective and efficient operating procedures for clinicians and hospitals. As summarized by the President of the University of Miami, "the time is right for technobiology breakthroughs". The two National Academies have since collaborated on a number of regional meetings to underscore the benefits of working together; indeed, on June 24, 2015, the two National Academies and Case Western Reserve University sponsored another regional meeting to discuss the advances of technology and medical science in the detection, prevention and treatment of concussion.

In order to further introduce our faculties to each other, we established a series of collaborative research exchange forums (CREFs). Each CREF involved a half day workshop, beginning in the morning with several up-to-30-minute presentations on a pre-identified topic area by faculty members who were seeking collaborative partners from the other academic unit. Each presentation was followed by a brief question-and-answer period, and lunch was served after the 
presentations with ad-hoc groups forming to discuss joint efforts for writing up-to-\$100K CREF proposals that, if selected through a peer review process, were jointly funded by MSoM and CoE. The purpose of the CREF efforts was to carry out pilot investigations which would lead to the development of formal NIH or NSF proposals for funding within the year. The CREF program was both popular and successful; indeed, we curtailed the program, after achieving several successes and after we realized that faculty from our two academic units had no difficulty in working with each other. Today, collaborative efforts between our two faculties are quite normal; more importantly the recruitment of new biotechnology and technobiology faculty, is being carried out with mutual consultation and support.

Table 7 Technobiology examples by focus

\begin{tabular}{|c|c|c|c|}
\hline Focus & Example & Purpose & Scope \\
\hline \multirow{9}{*}{$\begin{array}{l}\text { Infor- } \\
\text { mation }\end{array}$} & Smart phone app & Diagnostics \& medication management & Servgood \\
\hline & Electronic portal & Tracking care & Service \\
\hline & Co-produced healthcare & Comprehensive health care reform & Service \\
\hline & Evidence-based medicine & Better clinical care through knowledge & Service \\
\hline & Connected servgoods & Balance between security \& privacy & Service \\
\hline & Video conferencing & Expanding rural care & Servgood \\
\hline & Biostatistics & Risk analysis \& design of experiment & Service \\
\hline & Mass customization & Customized health insurance & Service \\
\hline & Machine learning & Reading CT \& colonoscopy scans & Service \\
\hline \multirow{9}{*}{$\begin{array}{l}\text { Instru- } \\
\text { menta- } \\
\text { tion }\end{array}$} & Fitness device & Tracking diet \& exercise & Servgood \\
\hline & Augmented reality & Real-time analysis \& recognition & Servgood \\
\hline & Prosthetics & Wearable bionics \& exoskeleton & Servgood \\
\hline & Imaging & Non-intrusive scanning (PET, MRI, CT) & Servgood \\
\hline & Personal electrocardiogram & Smart phone connected ECG & Servgood \\
\hline & Cinematic reality & 3-D imagery & Servgood \\
\hline & Fast DNA sequencing & Liquid biopsy (blood test for cancer) & Servgood \\
\hline & Personalized medicine & Tailoring through genetic profiling & Servgood \\
\hline & Genetic testing of biopsy & Oncotype DX prostrate cancer & Servgood \\
\hline \multirow{9}{*}{$\begin{array}{l}\text { Inser- } \\
\text { tion }\end{array}$} & DNA-editing & Genetically modified cells & Servgood \\
\hline & Cardiac pacemaker & Monitoring \& pacing heart beats & Servgood \\
\hline & Injectable biomaterials & Scaffolds \& myocardial infarctions & Servgood \\
\hline & Micro-robotic surgery & Less invasive \& more accurate & Servgood \\
\hline & Additive manufacturing & Regenerative medicine (body part) & Servgood \\
\hline & Anti-extravasate drugs & Blocking cancer cell metastasis & Servgood \\
\hline & Bioelectronics & Artificial retina & Servgood \\
\hline & Insulin pump & Controlling diabetes & Servgood \\
\hline & 10 -hour pillcam in colon & Camera pill captures intestinal images & Servgood \\
\hline
\end{tabular}

Another collaborative venue occurred when UM and real estate developer Wexford undertook a joint venture to build a Life Science Park that will eventually accommodate five, $250 \mathrm{~K}$ square-foot buildings, housing medically-related companies, science centers, and research institutes. While the first building was being completed in 2008, we were able to convince UM and Wexford to call it the Life Science and Technology Park, in recognition of the fact engi- 
neering and technology play an indispensable role in world-class discoveries and developments, especially within the healthcare domain. The now-renamed UM Life Science and Technology

Table 8 Technobiology research examples by CoE department

\begin{tabular}{|c|c|c|c|}
\hline Project Title & Department & Focus & Funding Agency \\
\hline Genetic regulation of adult stem cells & Biomedical & Insertion & Veteran Affairs \\
\hline Creating synthetic brain through hybrid computing & Biomedical & Information & SUNY Found \\
\hline Neural correlates of tourette syndrome & Biomedical & Instrumentation & NIND\&S \\
\hline Functionalized nanoscale coatings for islet encapsulation & Biomedical & Insertion & NI of Diabetes \\
\hline UM clinical and translational science institute (CTSI) & Biomedical & Information & NIH \\
\hline Identifying individuals using ear patterns & Electrical & Information & W Va Research \\
\hline Modeling, analysis \& simulation of gene networks & Electrical & Information & $\mathrm{NSF}$ \\
\hline Computationally-scalable tumor Markov models & Electrical & Information & InfoTech \\
\hline Acoustics lab on a chip for studies of tumor cells & Electrical & Information & $\mathrm{NSF}$ \\
\hline Optical coherence tomography for molecular eye imaging & Electrical & Information & NI on BMI \\
\hline Design for independent net-zero water buildings. & Civil \& Env & Instrumentation & NSF \\
\hline Center for tropical \& subtropical oceans \& human health & Civil \& Env & Instrumentation & $\mathrm{NSF}$ \\
\hline Relative assessment of human health \& ecological risks & Civil \& Env & Instrumentation & Caribbean Cruise \\
\hline Leachability \& harmful health effects of $\mathrm{As}, \mathrm{Cu}, \mathrm{Cr}$ & Civil \& Env & Instrumentation & FL State Health \\
\hline Pathogen monitoring in south Florida & Civil \& Env & Instrumentation & FL State Health \\
\hline Occupational \& environmental health risks in solid waste & Industrial & Information & Hinckley Center \\
\hline Primary care evaluation research & Industrial & Information & Health Fund \\
\hline Center on research \& education for aging \& technology & Industrial & Information & NI on Aging \\
\hline Medical reservist team training program & Industrial & Information & US Army \\
\hline Miami clinical and translational science institute & Industrial & Information & NIH \\
\hline Novel quantitative technology for tissue engineering & Mechanical & Instrumentation & NI on BMI \\
\hline Image-guided robotic orthopedic surgery & Mechanical & Instrumentation & Mako Surgical \\
\hline 3 -D printing of eye socket & Mechanical & Insertion & Private Foundation \\
\hline Internet-based visual rating \& image-based morphing & Mechanical & Instrumentation & Mount Sinai \\
\hline Flow diverters to cure cerebral aneurysms & Mechanical & Instrumentation & NI of ND\&S \\
\hline
\end{tabular}

Park (LSTP) serves as a collaborative center for UM's MSoM and CoE, as well as for other forward-looking enterprises, to undertake potentially life-enhancing work. Another major collaboration occurred when faculty from MSoM's Department of Pathology and the CoE partnered to establish the Biomedical Nanotechnology Institute at the University of Miami (BioNIUM), a LSTP facility that includes a S100 cleanroom and is being generously supported by a gift from the Dr. John T. Macdonald Foundation. In sum, we see the LSTP as an innovation gateway to life science and technology!

Other collaborative efforts are identified in Table 8 which lists example projects that our $\mathrm{CoE}$ faculty have undertaken in concert with their MSoM counterparts; the projects are grouped by the five $\mathrm{CoE}$ departments, along with each project's focus (i.e., information, instrumentation, or insertion) and funding agency. One project of note is the multi-year, multi-million dollar Miami Clinical and Translational Science Institute (CTSI); it seeks to assist researchers and clinical professionals through all phases of the process of translating research into meaningful 
health advances.

\section{Concluding Insights}

The previous sections have demonstrated the importance of considering healthcare from a systems engineering perspective, especially from a technobiology — some call it "e-health" [22] - perspective. In essence, healthcare is a people-intensive service system. Moreover, in order to be an efficient and effective system, it must be, respectively, an integrated and adaptive system, all of which adds to the complexity of the service system. As indicated throughout the paper, there are many areas where new and additional methods are required to define, model and solve an aspect of the technobiology-related healthcare system. Much research remains to be undertaken.

A number of other insights can be ascertained from an integrated and adaptive view of healthcare services. First, as noted earlier, information - especially in regard to electronicbased medical records - constitute the glue that should keep the healthcare system integrated and adaptive. Unfortunately, most information - including patient data, drug prescriptions, laboratory diagnostics, clinician reports, and body scans - are still in manual folders and, as a consequence, difficult to access, fuse and analyze. More recently, Microsoft and Google, respectively, launched HealthVault and Health for consumers to store and manage their personal medical data online, and Wal-Mart is allowing its intranet to serve as a repository for the health histories of its more than one million staff members. While patients have a legal right to obtain their medical records from doctors, hospitals, and testing laboratories, it would indeed be a tedious and overwhelming process if the records were not in electronic form. Nevertheless, sharing such electronic records with new medical providers and third-party services should make it easier to cut waste, eliminate red tape, coordinate care, spot adverse drug interactions, reduce repeat or ineffective tests, allow for medication reminders, and track vital signs. Indeed, as part of the 2009 economic stimulus package, the US is mounting a massive effort to modernize healthcare by making all health records standardized and electronic, a monumental task given that in 2009 only about $17 \%$ of the 800,000 clinicians employ computerized records. Moreover, personal data residing on Microsoft, Google or Wal-Mart server grids or clouds do raise significant privacy concerns. At present, the Health Insurance Portability and Accountability Act (HIPAA) only requires doctors, hospitals and third-party payers to not release information without a patient's consent. Of course, HIPAA's requirements could be broadened and new rules could be enacted that give consumers stronger protection and legal recourse if their records are leaked or improperly shared for other than its intended purpose. In a world where the Internet of Things or connected servgoods dominate - including the sharing of health-related data over social networks like Facebook, Twitter, Instagram and Snapchat the balance between security and privacy is becoming increasingly more complex ${ }^{[23]}$. The online world is populated with criminal enterprises that see value and opportunities in our identities, credit cards and other personal data.

Second, as real-time healthcare decisions must be made in an accelerated and co-produced manner, the human service provider (e.g., clinician) will increasingly become a bottleneck; he/she must be supported by a smart robot or software agent. For example, the clinician could 
use a smart alter ego or agent — sometimes called a virtual personal assistant — which could analyze, and perhaps fuse, all the existing and incoming e-mails, phone calls, Web pages, $\mathrm{x}$ rays, drug prescriptions, and medical examinations, and assigns every item a priority based on the clinician's preferences and observed behaviors. It should be able to perform an analysis of a message text, judge the sender-recipient relationships by examining an organizational chart and recall the urgency of the recipient's responses to previous messages from the same sender. To this, it might add information gathered by watching the clinician via a video camera or by scrutinizing his/her calendar. Most probably, such a smart agent would be based on a Bayesian statistical model - capable of evaluating hundreds of user-related factors linked by probabilities, causes and effects in a vast web of contingent outcomes - which can infer the likelihood that a given decision on the software's part would lead to the clinician's desired outcome. The ultimate goal is to judge when the clinician can safely be interrupted, with what kind of message, and via which device. In time, smart agents representing both providers and consumers will become the service co-producers; they will employ decision informatics and cloud computing techniques to accomplish their tasks. It should be noted that smart agents may never be appropriate for certain situations, especially, as examples, where a nuanced patient behavior is critical or when a catastrophic surgical consequence is on the balance. Obviously, these situations require direct patient-clinician interaction or co-production, perhaps assisted by smart agents - with access to databases and algorithms that are a part of cloud computing - which can help in the identification of alternate diagnoses and treatments.

Third, perhaps the best example of an integrated and adaptive service system is the evolving Web 2.0. It is user-built, user-centered and user-run. In other words, it is a social network for integration - including collaboration and communication — of activities (e.g., eBay, Amazon.com, Wikipedia, Twitter, etc.), entertainment (e.g., Facebook, Ning, Bebo, etc.), searches (e.g., Google, Yahoo, MSN.com, Bing, Bidu, etc.), and knowledge computation (e.g., Wolfram Alpha). Unfortunately, the integrated web, while being a somewhat successful e-commerce platform, is unable to interpret, manipulate or make sense of its content. On the other hand and with the encoding of web pages in a semantic web format, the evolving web will be able to allow for the above mentioned smart or decision informatics supported agents to undertake semantic analysis of user intent and web content, to understand and filter their meaning, and to adaptively respond in light of user needs. The Semantic Web, then, could be an ideal complex service system where integration and adaptation will constitute the basis for its functionality. However, several obstacles must be overcome before reaching full functionality. For example, semantic standards or ontologies - such as the Web Ontology Language (OWL) - must be established so as to maintain compatible and interoperable formats; at present, healthcare and financial services companies are each developing their own ontology. Indeed, a healthcare system of systems (SoS) also needs a common ontology to allow for new system components to be appropriately integrated into the SoS without a major effort, so as to achieve higher capabilities and performance than would be possible with the component systems being stand-alone systems. Of course, the healthcare ontology must be transdisciplinary — beyond a single disciplinary - in scope; with such an ontology, healthcare may indeed exist as a social network of patients, clinicians, insurers and other related providers. 
Fourth, as a critical aspect of complexity, modern systems of systems are also becoming increasingly more human-centered, if not human-focused; thus, services, goods and servgoods are becoming more personalized or customized. Certainly, services co-production implies the existence of a human customer, if not a human service provider. The implication is profound: A multidisciplinary approach must be employed for, say, healthcare - it must also include techniques from the social sciences (i.e., sociology, psychology, and philosophy) and management (i.e., organization, economics and finance). As a consequence, researchers must expand their systems (i.e., holistic-oriented), man (i.e., decision-oriented) and cybernetic (i.e., adaptiveoriented) methods to include and be integrated with those techniques that are beyond science and engineering. For example, higher patient satisfaction can be achieved not only by improving service quality but also by lowering patient expectation. In essence, as stated by Hipel et al. ${ }^{[24]}$, systems, man and cybernetics is an integrative, adaptive and multidisciplinary approach to creative problem solving; it takes into account stakeholders' value systems and satisfies important societal, environmental, economic and other requirements in order to enhance the decision making process when designing, implementing, operating and maintaining a system or system of systems to meet societal needs in a fair, ethical and sustainable manner throughout the system's life cycle. Interestingly, an adaptive, human-centered (i.e., human-to-human) system that functions in real-time is the Twitter social network, based on easy-to-use, 140-character bursts of constant chatter which can inform and engage a participant with an intensity that cannot be replicated offline.

Fifth, technology advances are facilitating the customization or personalization of medical treatments through advances in genetics, proteomics and metabolomics. Most common illnesses will eventually be preventable; the challenge is to know which prevention effort will be most effective for a given individual. Employing markers of risk (e.g., gene variants, blood levels of a protein moiety, etc.) may allow for the targeting or personalization of preventive measures in a highly cost-effective way. In this manner, humans can be sheltered from chronic illnesses and pandemics, and remain fully functional until an advanced age (say, 100), beyond which survival is genetically limited. Thus, healthcare is indeed a service, one that can be personalized and that can be employed to enhance the quality and length of an individual's life. On the other hand, embarking on such a germ-line gene therapy or editing the human genome will lead us ever closer to creating a genetically modified human, an outcome that many are unanimously against for both moral and ethical reasons. Opposition is also growing against the development of an autonomous non-human intelligence (i.e., artificial intelligence).

Sixth, a final insight concerns the possible integration or convergence of technology and biology, either through technobiology and/or through biotechnology. Interestingly, the University of Illinois at Urbana-Champaign has just recently announced that it will be opening a new engineering-based College of Medicine in Fall 2017, in partnership with the Carle Health System. The independently accredited medical school will seek to fuse engineering, technology, Big Data and medicine; in sum, it will seek to advance breakthroughs in both technobiology and biotechnology. 


\section{References}

[1] Ereky K. Biotechnology of meat, fat and milk production in an agricultural large-scale farm. Budapest, Hungary, 1919.

[2] World Health Organization Report (WHO). Why do health systems matter. Geneva, Switzerland: World Health Organization, 2000.

[3] Tien J M, Goldschmidt-Clermont P J. On designing an integrated and adaptive healthcare system. Engineering a Learning Healthcare System: A Look to the Future, Washington, DC: Institute of Medicine, 2009.

[4] National Academies. Engineering the health care system. Washington, DC: National Academies Press, 2006.

[5] Rouse W B. Health care as a complex adaptive system: Implications for design and management. National Academy of Engineering: The Bridge, 2008, 38(1): 17-25.

[6] Parasher A, Goldschmidt-Clermont P, Tien J M. Healthcare delivery as a service system: Barriers to co-production and implications of healthcare reform. Chapter 9 in Management Engineering for Effective Healthcare Delivery, Edited by Kolker A and Story P, 2012.

[7] US President. Executive order on critical infrastructure protection. Washington, DC: The White House, October 16, 2001.

[8] Tien J M, Cahn M F. An evaluation of the wilmington management of demand program. Washington, DC: National Institute of Justice, 1981.

[9] Tien J M, Krishnamurthy A, Yasar A. Towards real-time customized management of supply and demand chains. Journal of Systems Science and Systems Engineering, 2004, 13(3): 257-278.

[10] Goldschmidt-Clermont P J, Dong C, Rhodes N M, et al. Perspective: Autonomic care systems for hospitalized patients. Academic Medicine, 2009, 84(12): 1727-1731.

[11] Tien J M. Towards a decision informatics paradigm: A real-time, information-based approach to decision making. IEEE Transactions on Systems, Man, and Cybernetics, Special Issue, Part C, 2003, 33(1): 102-113.

[12] Kaplan E H, Craft D L, Wein L M. Emergency response to a smallpox attack: The case for mass vaccination. Proceedings of the National Academy of Sciences, 2002, 99(16): 10935-10940.

[13] Tien J M, Berg D. A case for service systems engineering. Journal of Systems Science and Systems Engineering, 2003, 12(1): 13-38.

[14] Tien J M. Internet of connected servgoods: Considerations, consequences and concerns. Journal of Systems Science and Systems Engineering, 2015, 21(4): 385-415.

[15] Porter H E, Hippelmann J E. Managing the Internet of Things: How smart, connected products are changing the competitive landscape. Boston, MA: Harvard Business School Press, 2014.

[16] Massachusetts Institute of Technology (MIT). Top 50 smartest companies. Technology Review, February, 2014.

[17] Institute of Electrical and Electronics Engineers (IEEE). Hacking the human OS: How big data will transform medicine and health. Piscataway, NJ: IEEE Spectrum: Special Report, July, 2015.

[18] Taleb N N. The black swan. New York, NY: Random House, Inc, 2010.

[19] Tien J M. Overview of Big Data: A US perspective. National Academy of Engineering: The Bridge, 2014, 44(4): $12-19$.

[20] Carr N. The shallows: What the internet is doing to our brains. New York, NY: Norton, 2010.

[21] Tien J M, Goldschmidt-Clermont P J. Healthcare: A complex service system. Journal of Systems Science and Systems Engineering, 2009, 18(4): 285-310.

[22] Michel H E. E-health: Our new endless frontier. IEEE: The Institute, June, 2015, 13.

[23] Goodman M. Future crimes. New York, NY: Doubleday, 2015.

[24] Hipel K W, Jamshidi M M, Tien J M, et al. The future of systems, man and cybernetics: Application domains and research methods. IEEE Transactions on Systems, Man, and Cybernetics Part C, 2007, 30(2): $213-218$. 